\title{
Efectividad de un programa de alta resolución ambulatoria gracias al uso de la Ecografía Urológica
}

\section{Effectiveness of a high resolution program outpatient thanks to the use of Ultrasound Urological}

\author{
Laura Redón-Gálvez ${ }^{1}$ Manuel Ramón Diz Rodriguez ${ }^{1}$ Carlos Martín-García ${ }^{1} \quad$ Vicente Chiva-Robles ${ }^{1}$ \\ Olivia Matos-Orozco ${ }^{1}$ Marcos Lujan-Galán ${ }^{1}$
}

${ }^{1}$ Department of Urology, Hospital Infanta, Cristina, Parla, Madrid, Spain

Address for correspondence Laura Redón-Gálvez, Camino del Molino Urol Colomb 2020;29:231-234.

N2, Madrid, España (e-mail: laureixon@hotmail.com).

\section{Resumen \\ Palabras clave \\ - ecografía \\ - alta resolución \\ - factores sociodemográficos- estructurales relacionados}

\section{Abstract}

Objetivos Estimar la efectividad de la ecografía en consultas externas de alta resolución de urología. Como objetivos secundarios, se evaluó el impacto de otros factores como la edad, sexo, prioridad y procedencia de la consulta.

Métodos Se analizó retrospectivamente a pacientes que acudieron a una primera visita a la consulta de urología del hospital entre los años 2008-2018.

Se recogieron las variables edad, sexo, procedencia, prioridad de la consulta y realización de ecografía.

Realizamos un análisis descriptivo de aquellos pacientes que resultaron ser alta resolución y de aquellos en los que se había realizado una ecografía. Posteriormente realizamos un análisis univariado mediante el test de chi-cuadrado y multivariado mediante la regresión logística.

Resultados Se incluyeron 32980 primeras visitas, con una edad media en la primera visita de 48,2 años (mediana 48,2) y tratándose en la mayoría de hombres $(74,4 \%$ ). La proporción de pacientes que pudieron ser dados de alta tras una primera visita, fue del 16\% y gracias a la realización de una ecografía llegó al 16,7\%.

En el análisis univariado, tanto la procedencia, la prioridad, la ecografía realizada, el sexo y la edad, se asociaron a que la consulta realizada fuera de alta resolución. En el análisis multivariado, se confirmó la asociación de todas las variables (exceptuando la prioridad, $\mathrm{p}=0,37$ ), aumentando el riesgo de modo discreto (entre $0,3-0,9$ veces).

Conclusiones La ecografía resultó ser una buena herramienta en las consultas de alta resolución, llegándose a realizar hasta casi en un $20 \%$ de los casos. Sin embargo, aunque tanto la ecografía como la edad, sexo y procedencia son factores relacionados con la alta resolución, esa relación no es relevante desde un punto de vista clínico.

Objectives Estimate the effectiveness of ultrasound of ultrasound in high resolution urology outpatients. As secondary objectives, the impact of other factors such as age, sex, priority and origin of the consultation was evaluated. received

June 30,2020

accepted

August 31, 2020
DOI https://doi.org/

10.1055/s-0040-1721324.

ISSN 0120-789X.

e ISSN 2027-0119.
Copyright (c) 2020, Thieme Revinter Publicações Ltda.., Rua do Matoso 170 , Rio de Janeiro, RJ, CEP 20270-135, Brazil. Todos los derechos reservados.

\section{License terms}

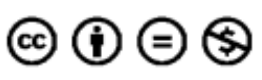




\section{Keywords}

- ultrasound

- high resolution

- related sociodemographicstructural factors
Methods Patients who attended a first visit to the hospital's urology consultation between 2008-2018 were retrospectively analyzed.

The variables age, sex, origin, priority of the consultation and ultrasound were collected. We performed a descriptive analysis of those patients who turned out to be high resolution and of those in whom an ultrasound had been performed. Subsequently, we performed a univariate analysis using the chi-square test and a multivariate analysis using logistic regression.

Results A total of 32,980 first visits were included, with a mean age at the first visit of 48.2 years (median 48.2 ) and being treated in the majority of men (74.4\%).

The proportion of patients who could be discharged after a first visit was $16 \%$, and thanks to an ultrasound it was $16.7 \%$.

In the univariate analysis, both the origin, priority, ultrasound performed, sex and age were associated with the consultation being of high resolution. In the multivariate analysis, the association of all variables was confirmed (except for priority, $p=0.37$ ), increasing the risk discretely (between 0.3-0.9 times).

Conclusions Ultrasonography turned out to be a good tool in high-resolution consultations, with up to $20 \%$ of cases being performed. However, although both ultrasound and age, sex and provenance are factors related to high resolution, this relationship is not relevant from a clinical point of view.

\section{Introducción}

El Sistema Nacional de Salud (SNS) en España sufre una enorme presión asistencial. En 2018 se calculó que se habían producido un total de 234000 millones de visitas al año, lo que corresponde a 5,1 visitas/habitante/año. ${ }^{1}$

Esos datos promueven la existencia de la llamada consulta de alta resolución (AR), que consiste en una visita ambulatoria donde se realizan en un mismo acto, todas las pruebas necesarias para el diagnóstico y tratamiento del paciente. ${ }^{2}$

Ese tipo de consultas tiene sus ventajas y sus inconvenientes. Las ventajas son principalmente para los pacientes ya que ahorran tiempo, disminuyen la ansiedad al saber el diagnóstico en el mismo día, y es un único especialista el que se ocupa de su problema. En cambio, supone cambios importantes en el servicio médico: las unidades de diagnóstico deben estar permanentemente disponibles, los médicos deben acostumbrarse a pensar rápidamente para poder dar un diagnóstico en el momento, y el personal de las consultas deben tener una gran versatilidad de forma que incluso las enfermeras puedan realizar pruebas diagnósticas. ${ }^{3,4}$

En el ámbito de la urología las principales pruebas diagnósticas son la ecografía y la cistoscopia, pruebas que casi todos los servicios de urología tienen disponibles en la actualidad. $^{5}$ En nuestro servicio, la principal prueba diagnóstica de cara a la AR es la ecografía, la cual tiene una gran versatilidad y nos permite realizar un enfoque diagnóstico-terapéutico en el mismo momento en que se está realizando.

El objetivo principal del estudio fue estimar la efectividad de la ecografía en consultas externas de alta resolución de urología. Como objetivos secundarios, se evaluó el impacto de otros factores como la edad, el sexo, la procedencia y prioridad de las consultas.

\section{Métodos}

Se analizó retrospectivamente a pacientes que acudieron a una primera visita a la consulta de urología del hospital Infanta Cristina (Parla, Madrid), entre los años 2008-2018. Se excluyeron las consultas que eran de revisión de patología ya atendida en fechas previas.

Se realizó ecografía abdominal a los pacientes que acudieron por hematuria micro o macroscópica o cólico nefrítico; y con ecografía testicular, a los que presentaban dolor o sensación de tumoración testicular. En el caso de la ecografía testicular, pudo realizarse consulta de alta resolución si los hallazgos fueron normales pero no si se detectó un tumor testicular o un proceso beningo (hidrocele, quiste...) que requiriera intervención quirúrgica. En el caso de la ecografía renal por cólico nefrítico pudo darse de alta aquellos pacientes donde la ecografía no se detectó patología, pero no en aquellos donde se detectó hidronefrosis o litiasis porque se solicitó un escáner para completar el estudio. Y por último, en el caso de la ecografía renovesical por hematuria macroscópica o microscópica no pudo realizarse consulta de alta resolución en ningún caso porque en esos procesos, está indicado completar el estudio con otras pruebas complementarias (cistoscopía, citología +/-) a pesar de que la ecografía sea normal.

La ecografía fue realizada por un urólogo o una enfermera con formación previa en ecografía ya que ambos habían realizado el Curso básico de ecografía urológica para urólogos, médicos de familia y personal de enfermería, curso auspiciado por la Asociación Española de Urología. 
Se recogieron las variables edad, sexo, procedencia (hospital o atención primaria), prioridad de la consulta (normal o preferente) y realización de ecografía.

Realizamos un análisis descriptivo de aquellos pacientes que resultaron ser AR y de aquellos en los que se había realizado una ecografía. Posteriormente realizamos un análisis uni y multivariante mediante el test de chi cuadrado y test de Mann-Whitney, así como regresión logística tipo "backward stepwise", para identificar las variables relacionadas con la $\mathrm{AR}$.

El nivel de significación estadística se estableció en $\mathrm{p}<0,05$. El manejo de los datos se realizó mediante la aplicación MS Access, y para el análisis estadístico se empleó el paquete IBM-SPSS 22.0.

\section{Resultados}

Se incluyeron 32980 primeras visitas, con una edad media en la primera visita de 48,2 años (mediana 48,2 ) y tratándose en su mayoría de hombres $(74,4 \%)$.

La mayoría de los pacientes procedían de atención primaria (69,5\%) y tenían una prioridad no preferente (91\%).

La proporción de pacientes que pudieron ser dados de alta tras una primera visita fue del $16 \%$ y gracias a la realización de una ecografía fue del $16,7 \%$.

En cuanto a los factores que influyeron en la realización de una consulta de AR, esos fueron en el análisis univariado, la procedencia de atención primaria, la prioridad normal, la realización de una ecografía, el sexo masculino y la edad $<50$ años ( $\mathrm{p}<0,001$ para todos los parámetros estudiados). - Tabla 1. En el análisis multivariado se confirmó la asociación de los distintos factores de un modo discreto (procedencia RR 0,673 IC 95\% 0,627-0,722, ecografía RR 0,388 IC 95\% 0,356-0,24, sexo RR 0,55 IC 95\% 0,508-0,596 y edad RR $0,987$ IC $95 \%$ 0,986-0,989), exceptuando la prioridad ( $\mathrm{p}=0,37)$.

- Tabla 2
Tabla 2 Análisis multivariado de los posibles factores asociados con la AR

\begin{tabular}{|l|l|l|l|}
\hline VARIABLES & \multirow{2}{*}{ RR } & \multicolumn{2}{|l|}{ 95\% C.I. para RR } \\
\cline { 3 - 4 } & & Inferior & Superior \\
\hline Procedencia & 0,673 & 0,627 & 0,722 \\
\hline Ecografía & 0,388 & 0,356 & 0,423 \\
\hline Sexo & 0,55 & 0,508 & 0,596 \\
\hline Edad & 0,987 & 0,986 & 0,989 \\
\hline
\end{tabular}

\section{Discusión}

Como técnica de imagen, la ecografía tiene una serie de características únicas: portátil, no radioactiva, poco costosa, se puede repetir múltiples veces y muestra la imagen en tiempo real. ${ }^{6}$

Precisamente por esos motivos, es una técnica de imagen que se está utilizando cada vez con mayor asiduidad en las consultas de AR. Ejemplo de ello lo tendríamos en el estudio de Jones Ky col., ${ }^{7}$ en el que a un total de 93 mujeres que acudieron por un sangrado anormal uterino se les practicó una ecografía transvaginal, logrando diagnosticar a un $89,2 \%$ en una única visita. Otro ejemplo de ello lo tendríamos en el estudio de Sinha R y col., ${ }^{8}$ donde se practicó una ecografía y posterior biopsia guiada por ecografía, en pacientes que acudían por una tumoración en la piel. Eso permitió en algunos casos, ahorrarse la realización de una resonancia magnética, pudiendo diagnosticarse un sarcoma en una primera visita.

En nuestro estudio, la ecografía resultó ser una buena herramienta en las consultas de AR, llegándose a realizar hasta en un $20 \%$ de los casos.

Revisando la literatura, existen muy pocos estudios que analicen los factores que pueden influir en la realización de

Tabla 1 Análisis univariado de los posibles factores asociados con AR

\begin{tabular}{|c|c|c|c|c|}
\hline \multirow[t]{2}{*}{ VARIABLES } & \multirow[t]{2}{*}{ Categorías } & \multicolumn{2}{|c|}{ ALTA RESOLUCIÓN } & \multirow[t]{2}{*}{$P$} \\
\hline & & NO & sí & \\
\hline \multirow[t]{2}{*}{ Sexo } & Mujer & 6861 (89\%) & $859(11 \%)$ & \multirow[t]{2}{*}{$<0,001$} \\
\hline & Varón & $20831(82,4 \%)$ & $4438(17,6 \%)$ & \\
\hline \multirow[t]{2}{*}{ Edad } & $<50$ años & $24395(88,09 \%)$ & $4126(11,91 \%)$ & \multirow[t]{2}{*}{$<0,001$} \\
\hline & $\geq 50$ años & $3297(87,05 \%)$ & $1162(12,95 \%)$ & \\
\hline \multirow[t]{2}{*}{ Procedencia } & Hospital & $8853(87,9 \%)$ & $1213(12,1 \%)$ & \multirow[t]{2}{*}{$<0,001$} \\
\hline & Atención primaria & $18839(82,2 \%)$ & $4075(17,8 \%)$ & \\
\hline \multirow[t]{2}{*}{ Prioridad } & Normal & $25077(83,6 \%)$ & $4927(16,4 \%)$ & \multirow[t]{2}{*}{$<0,001$} \\
\hline & Preferente & $2615(87,9 \%)$ & $361(12,1 \%)$ & \\
\hline Ecografía & No & $25595(85,3 \%)$ & $4403(14,7 \%)$ & \multirow[t]{2}{*}{$<0,001$} \\
\hline Realizada & Sí & 2097 (70,3\%) & $885(29,7 \%)$ & \\
\hline
\end{tabular}

En negrita los factores asociados con la misma, en conclusión, todos ellos. 
consultas de alta resolución. En el estudio de Zambrana y col., 9 se subraya que principalmente se necesitan dos elementos estructurales: una buena coordinación con atención primaria con la existencia de protocolos que permitan que el paciente sea remitido con pruebas complementarias básicas, y contar con equipamiento médico adecuado, en nuestro caso la ecografía.

Además, pueden influir tanto factores demográficos de los pacientes (la edad y el sexo), como características de la propia consulta (prioridad y procedencia) como la disponibilidad para la realización de ecografía in situ como comentamos previamente.

En el estudio de Páez y col., ${ }^{3}$ se subraya que las consultas de AR son más frecuentes en varones jóvenes, como también ocurre en nuestro estudio, planteándose que probablemente sea debido a que los varones jóvenes consultan por motivos más sencillos en los que solo es necesario un abordaje empírico o requieren de la utilización de una prueba diagnóstica simple como es la ecografía, y eso ocurriría por ejemplo, en los casos de dolor testicular.

En cuanto a las características de la consulta, si hablamos de la procedencia en nuestro estudio, se realizaron más visitas de AR en los pacientes que procedían de atención primaria que en los que procedían del hospital, sin poder explicar dicha relación ni encontrar referencias al respecto en la literatura.

Hay que tener en cuenta que todos esos aspectos que hemos visto relacionados con la AR (edad y sexo de los pacientes, prioridad y procedencia de la consulta y realización de ecografía), no fueron relevantes desde un punto de vista clínico en nuestro estudio, por lo que en un futuro sería interesante realizar más estudios donde se demostrara con robustez dicha relación.

\section{Conclusiones}

La ecografía resultó ser una buena herramienta en las consultas de AR, llegándose a realizar hasta casi en un
$20 \%$ de los casos. Sin embargo, aunque tanto la ecografía como la edad, sexo y procedencia son factores relacionados con la AR, esa relación no es relevante desde un punto de vista clínico.

Conflicto de Intereses

Los autores declaran no tener ningún conflicto de intereses.

\section{Referencias}

1 Sistema Nacional de Salud. Actividad ordinaria en Atención Primaria del Sistema Nacional de Salud (SNS), consultas médicas atendidas en los centros y domicilios y frecuentación general anual según comunidad autónoma. 2018

2 Falces C, Sadurní J, Monell J, et al. [One-stop outpatient cardiology clinics: 10 years' experience]. Rev Esp Cardiol 2008;61(05): 530-533

3 Páez A, Redondo E, Sáenz J, Marín M, Juárez N, Durán M. The onestop clinic as the standard of out-patient care in a hospital urology department. Int Braz J Urol 2011;37(05):623-629

4 Queipo Zaragozá JA, Moral Baltuille A, Beltrán Meseguer JF, et al. La asistencia urológica en el Centro Sanitario Integral "Alto Palancia": modelo de integración primaria-especializada y consulta única. Actas Urol Esp 2005;29(03):305-310

5 Coull N, Rottenberg G, Rankin S, et al. Assessing the feasibility of a one-stop approach to diagnosis for urological patients. Ann R Coll Surg Engl 2009;91(04):305-309

6 Celebi N, Griewatz J, Malek NP, et al. Development and implementation of a comprehensive ultrasound curriculum for undergraduate medical students - a feasibility study. BMC Med Educ 2019;19(01):170

7 Jones K, Bourne T. The feasibility of a 'one stop' ultrasoundbased clinic for the diagnosis and management of abnormal uterine bleeding. Ultrasound Obstet Gynecol 2001;17(06): 517-521

8 Sinha R, Mohamed AM, Karsandas A. The impact of ultrasound in an integrated one-stop sarcoma clinic. Clin Radiol 2020;75(04): 321.e21-321.e28

9 Zambrana-García JL, Torres-Jiménez M, Rubio-Sánchez JM, Montijano-Cabrera A, Peña-Ojeda JA, Velasco-Malagón MJ. Procesos médicos susceptibles de alta resolución en consultas ambulatorias. Rev Calid Asist 2017;32(02):82-88 